\title{
Robust Localization in Wireless Sensor Networks through the Revocation of Malicious Anchors
}

\author{
Satyajayant $\mathrm{Misra}^{\dagger}$, Guoliang Xue ${ }^{\dagger}$ and Aviral Shrivastava ${ }^{\dagger}$
}

\begin{abstract}
In a wireless sensor network (WSN), the sensor nodes (SNs) generally localize themselves with the help of anchors that are pre-deployed in the network. Time of Arrival (ToA) is a commonly used mechanism for SNs localization in WSNs. In ToA, the SNs localize themselves using the positions of the anchors and the time difference between the receipt of a radio and ultrasound signal transmitted by each anchor. In this setting, the localization process has a high risk of being subverted by malicious anchors that lie about their position and/or distance from the SNs. In this paper, we propose an efficient scheme that helps identify and revoke these malicious anchors. We use a mobile verifier (MV) that moves throughout the network, in some pre-determined manner, and obtains multiple location references from each anchor. For each anchor, the MV tests the mean and the variance of the collected sample to identify if the anchor is malicious. We show through simulations that our scheme successfully identifies more than $80 \%$ of malicious anchors with less than 60 references from each. Also, the percentage of false positives is close to 0 .
\end{abstract}

\section{INTRODUCTION}

Large scale distributed wireless sensor networks (WSNs) have become popular in both the military and civilian domains because of their infrastructureless nature and relative ease of deployment [1]. However, there still exist many fundamental problems that need to be addressed [7]. The problem of robust localization of the wireless nodes is one such fundamental problem in a WSN. Accurate localization is also very important because most applications require the position of the source of the data for effective utilization. In an infrastructureless WSN, for cost effectiveness, not all nodes are equipped with self-localizing abilities. Most sensor nodes (SNs) localize themselves using the position estimates of a group of nodes in the network called the anchors [10], [11], [13]. The anchors are fixed wireless nodes that know their own positions accurately, either through GPS or from pre-programmed information.

In this paper, we assume that Time of Arrival (ToA) [15], [13] is the underlying mechanism used for localization. Following the ToA method, each anchor $a_{i}$ periodically broadcasts its identifier (ID) and position information in its neighborhood, as a radio signal (RS) and an ultrasound signal (US) at the same instance of time. We denote these

This research was supported in part by ARO grant W911NF-04-1-0385 and NSF grants CNS-0524736 and CCF-0431167. The information reported here does not reflect the position or the policy of the federal government.

${ }^{\dagger}$ All three authors are with the Department of Computer Science and Engineering, Arizona State University, Tempe, AZ 85287-8809. Email: \{satyajayant, xue, aviral.shrivastava\}@asu.edu. two components together as the location reference. On receipt of the location reference $l_{i}$ from $a_{i}$, each sensor node $(\mathrm{SN}) u$, calculates the time difference in receipt of the signals and uses the constants, speed of light $(c)$ and sound $(s)$, to obtain an estimate $\hat{d}_{i}$ of its distance $\left(d_{i}\right)$ from $a_{i}$. The calculation of the estimate $\hat{d}_{i}$ is given below by Equations 1 and 2 as,

$$
\begin{array}{r}
\Delta t=\hat{d}_{i} / s-\hat{d}_{i} / c, \\
\hat{d}_{i}=\Delta t \cdot \frac{1}{1 / s-1 / c},
\end{array}
$$

where $\Delta t$ refers to the difference in time between the receipt of the RS and the US. We note that the wireless medium is inherently error-prone, hence the value of $\Delta t$ is inaccurate. This results in a SN $u$ being able to obtain only an estimate of $d_{i}$.

When $u$ gets a sufficient number of location references from anchors in its vicinity it can use them to estimate its own position. The estimation can be done using the Minimum Squared Error (MSE) (also referred to as the minimum mean square error) method [15], [10] given by,

$$
f=\min \sum_{i=1}^{n}\left(\left\|\hat{\mathbf{u}}-\mathbf{a}_{\mathbf{i}}\right\|-\hat{d}_{i}\right)^{2}
$$

where $\hat{\mathbf{u}}$ is an estimate of the real position $\mathbf{u}=\left(u_{x}, u_{y}\right)$ of $u, \mathbf{a}_{\mathbf{i}}=\left(a_{i x}, a_{i y}\right)$ is the position of anchor $a_{i}, \hat{d}_{i}$ is the estimate of the distance of $a_{i}$ from $u$ calculated by $u$ using the ToA method, and $\|\cdot\|$ is the Euclidean norm. $n$ is the number of anchors from whom $u$ receives the localization information. In the absence of measurement errors, $\hat{\mathbf{u}}$ is the correct estimate, that is, $\|\hat{\mathbf{u}}-\mathbf{u}\|=0$. In the presence of measurement errors, the error in $\hat{\mathbf{u}}$ is dependent on the measurement error. In this scenario, accurate localization is fairly complex as it is difficult to bound the estimation error. Given the complexity in accurate localization, the presence of malicious lying anchors makes accurate localization significantly more difficult to achieve. We demonstrate this with simulation results.

Problem Motivation: In our simulation set-up, each malicious (lying) anchor lied such that its distance from a SN in its range is between $[d, d \cdot(1+\epsilon)]$, where $d$ is the real distance and $\epsilon=0.5$. Figure 1(a) shows the average of the square of the error $\left(S_{\text {err }}\right)$ in localization over 20 iterations, when lying anchors are included in the localization process. Figure 1(b) shows $S_{e r r}$ when the lying anchors are not included in the localization process. We would like the reader to note the 


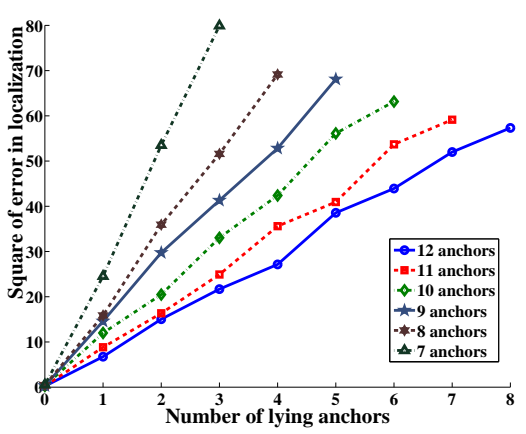

(a) Malicious anchors included in localization

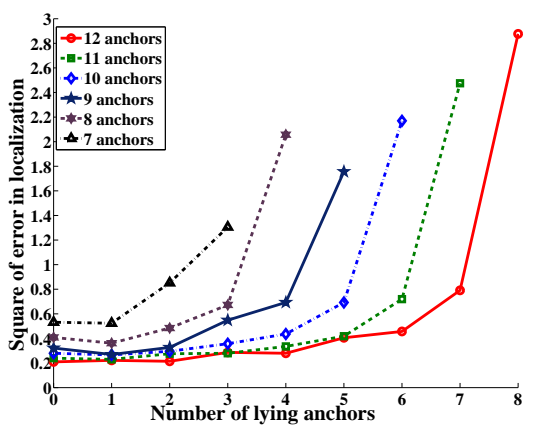

(b) Only true anchors used for localization

Fig. 1. Localization error in MSE method

difference in scale of the Y-axis in the two figures and point out that the value of $S_{e r r}$ when the number of lying anchors is 0 is the same in both cases. It is easy to see that the error in localization when malicious anchors are included is an order of magnitude higher than when the localization is done with only the true anchors. For instance, when there are 10 anchors in the range of a SN and 5 of them are malicious, the inclusion of malicious anchors in localization results in a value of $S_{\text {err }}>50$ sq.m. However, when localization is done without the malicious anchors, the value of $S_{\text {err }}<0.8$ sq.m. Thus, we can conclude that the presence of malicious anchors is detrimental to accurate localization and their revocation is necessary in the interest of increasing the accuracy.

In this paper, we propose a technique for identifying and revoking the malicious anchors in a WSN. In our scheme, we use a mobile verifier (MV), sent by the base station (BS), that obtains location references from the anchors and identifies the malicious ones by performing statistical analyses of the location references. The malicious anchors, once identified, can be revoked from the network, to prevent subversion of localization. Using our technique we could successfully identify and revoke more than $80 \%$ of the malicious anchors in the network using only 60 references from each anchor.

In Section II, we present related work. In Section III, we present the system and threat models along with their assumptions. Section IV presents our proposed mechanism, while Section V presents the simulation results. We conclude our paper in Section VI.

\section{RELATED WORK}

Localization schemes in WSNs may be classified as range-based or range-free. The range-based mechanisms, as proposed in [3], [16], [10], [5], perform localization by measuring properties such as point-to-point distance or angle estimates, whereas the range-free localization mechanisms as proposed in [8], [9], [11], [6], [15] do not require any physical measurements to perform localization. These mechanisms may use hop count or area-based estimation to localize a node [6]. Generally, range-based mechanisms lead to more accurate localization; however, they tend to be resource intensive and may require specialized hardware [16], [13]. The method used for position estimation is either based on minimum mean/median square estimation [15], [10], convex programming [4], [2], or triangulation [16].

There are many schemes, such as [5], [16], [10], [11], [8], [9], [11] that have been proposed to increase security and robustness of localization by either performing secure localization, doing location anomaly detection, or through location verification. Accurate localization in the presence of malicious anchors transmitting erroneous estimates has been dealt with by $\mathrm{Li}$ et al. [10], Liu et al. [11], and Du et al. [5]. In [10], [5], the schemes attempt to identify the anomaly and perform compromise resistant localization, whereas [11] attempts to detect and remove the malicious anchors from the network. However, the performance of the above schemes is severely limited in the presence of a large number of malicious anchors which may or may not be colluding. In this paper, we propose a novel scheme that can identify a large proportion of the malicious anchors even when the majority of the anchors in the network are malicious and colluding. This is possible because each anchor is verified independently, and hence the results of the verification of an anchor cannot be influenced by another.

\section{System Model AND Assumptions}

The system model used for our proposed technique is given below:

- The network consists of a set of anchors $\mathcal{A}=\left\{a_{i}, i=\right.$ $1, \ldots, n\}$ and a set of sensors $\mathcal{S}=\left\{s_{i}, i=1, \ldots, n\right\}$ that are deployed randomly and are fixed after deployment.

- Each anchor $a_{i}$ knows its own position $a_{i}\left(a_{i}=\right.$ $\left.\left(a_{i x}, a_{i y}\right)\right)$.

- The transmission range of the SNs is $r$ and the anchors is $R(R \geq r>0)$, while the reception range of the MV is $\geq R$.

- The anchors are equipped with radio/ultrasound transmitters and can transmit both signals simultaneously.

- The anchors broadcast their location references periodically. 
- The SNs and the MV are equipped with both radio and ultrasound receivers.

- The Mobile Verifier (MV) is GPS enabled and can obtain its own position accurately.

- Localization is required throughout the network's lifetime to localize new SNs added to the network.

- $\tilde{\mathbf{n}}$, the measurement error, is distributed normally, $\tilde{\mathbf{n}} \backsim$ $N\left(0, \sigma_{0}^{2}\right)$, with domain $x \in\left[-\delta_{\max }, \delta_{\max }\right]$. Thus, the probability $\operatorname{Pr}\left[\mathbf{n}<-\delta_{\max }\right]=\operatorname{Pr}\left[\mathbf{n}>\delta_{\max }\right]=0$. If $f(x)$ is the probability density function for a random variable $\mathbf{x} \sim N(0,1)$ then $\sigma_{0}^{2}$ is given by,

$$
\sigma_{0}^{2}=\frac{\int_{\delta_{\max }}^{-\delta_{\max }} x^{2} f(x) d x}{1-\int_{-\infty}^{-\delta_{\max }} f(x) d x-\int_{\delta_{\max }}^{\infty} f(x) d x}
$$

- The anchors transmit their references encrypted using a key from a hash chain. The key is released at a later time instant (delayed key disclosure mechanism). This is similar to the mechanism of $\mu$ TESLA [14].

- All devices have omnidirectional antennas.

\section{A. Assumptions}

- We assume that the anchors lie such that the resulting distance estimate is proportional to their actual distance from a $\mathrm{SN}$. The proportion is a uniform random variable $\sim U\left[-\epsilon_{\max }, \epsilon_{\max }\right]$, where $\epsilon_{\max }$ is a constant.

- No two anchors have the same position in the network.

- The anchors have enough memory and computation abilities to generate and store hash chains of the keys.

\section{B. Threat Model and Security Assumptions}

We assume that the malicious anchors may be compromised by a powerful external adversary to lie about their distance references. Also, the MV is assumed to be uncompromisable by an adversary. The MV could be a mobile vehicle or a human operator. In this subsection, we use $a_{i}$ to illustrate a malicious anchor.

The use of delayed key disclosure by the anchors for transmitting their location references ensures that malicious anchors in the neighborhood cannot change or replay the references. In addition, a malicious anchor $a_{i}$ cannot revoke a true anchor $a_{j}$ by masquerading as $a_{j}$ and broadcasting false location references. Our scheme is not affected by wormhole attacks. The MV can successfully identify wormhole attacks. For instance, if $a_{i}$ replays the location information of some other anchor $a_{j}$ not in the neighborhood, the MV can identify that $a_{j}$ does not belong to the neighborhood by analyzing the location references of $a_{j}$. In addition, $a_{i}$ can also be identified as the source of the message, since the MV can estimate the position $a_{i}$ from any 4 location references. We would like to note here that in ToA 4 references are required to estimate the position of a node [15].

In this setup, there are only three possible mechanisms by which a malicious anchor can subvert accurate localization, namely by lying about its position, its distance (by not transmitting the RS and the US simultaneously), or by lying both ways. If malicious anchor $a_{i}$ lies about its position so that the position changes but its ID remains same, then it can be easily caught by the SNs themselves. However, if $a_{i}$ uses a set of IDs, then the SNs will not be able to identify such an attack. This kind of attack can be identified by the MV. If the MV obtains 4 readings for each of the IDs used by $a_{i}$, it can easily estimate the location of the source $\left(a_{i}\right)$ and thus identify that the source of all the localization messages with different IDs to be $a_{i}$. Once $a_{i}$ is identified it may be revoked.

If $a_{i}$ lies by sending the RS and US at different times it could successfully cause distance reduction (US is sent earlier than the RS) or enlargement (US is sent later than the RS) attacks. Owing to the uncertainties due to measurement errors, this attack is difficult to identify by simply checking a few location references from a malicious anchor. Our technique identifies the malicious anchors in this scenario. The technique can also identify $a_{i}$, if it lies about its position and distance simultaneously (or even its position only). The reasons for the applicability of our scheme for all three scenarios is presented in the next section. We note here that distance enlargement/reduction attacks may also be caused by denial of service attacks. This kind of attack may be prevented by using error correcting codes or spread spectrum techniques described in literature [17]. We do not consider this attack in our threat model.

\section{Description OF THE SCHEME}

The MV is sent into the network by the Base Station (BS). The MV can be sent into the network any number of times. Each instance that the MV enters the network and returns to the $\mathrm{BS}$ is termed an iteration. In each iteration the MV obtains a constant number of location references $(\mathrm{K})$ from each anchor. $\mathrm{K}$ is a tunable system parameter which is dependent on the amount of energy required by the MV to obtain $\mathrm{K}$ references from each anchor, the amount of energy at the disposal of the MV, and also the desired level of accuracy. The MV may be recharged on getting back to the BS. In order to identify malicious anchors with high confidence, a MV has to obtain at least a certain number of location references from each anchor (derived later). The number of iterations is dependent on the total number of references required and the value of $\mathrm{K}$.

For our technique to be robust and efficient we need to address the following four questions, namely:

- How to ensure that all anchors are covered by the MV?

- How to make the route of the MV in the network appear random to an outside observer?

- How to perform statistical testing on the location references obtained from each anchor?

- How to revoke the anchors identified as malicious? 
We overlay the network with a virtual grid $(G r)$ of squares of side $R / \sqrt{2}$, where $R$ is the reception range of the MV. Each square in the grid is defined as $S_{x y}$ where $x$ and $y$ are the X-coordinate and Y-coordinate of the lower left corner of the square. The network may be represented by a graph $G(V, E)$, where $V=\left\{S_{i j}: S_{i j}\right.$ is a square in $\left.G r\right\} \cup B S$, $B S$ is the position of the BS, and $E=\left\{\left(S_{i j}, S_{k l}\right)\right.$ : $S_{i j}$ and $S_{k l}$ are adjacent $\}$. We note here that two vertices $S_{x y}$ and $S_{a b}$ are adjacent iff $S_{x y} \bigcap S_{a b} \neq \phi$, for the squares $S_{x y}$ and $S_{a b}$. In each iteration, the MV visits all the squares before returning to the BS, thus covering all the anchors.

\section{A. Setup of the paths of the $M V$}

The MV collects data passively in the network, thus its chance of getting detected is small. However, it is still possible for a strong external adversary to identify the presence of the MV in the network and learn its path. If the MV follows the same path in the network for each iteration, from the current position of the MV in a square $S_{i j}$, the adversary can identify the subsequent squares the MV will visit. It can then direct the malicious anchors in those squares to transmit correct location references during the time period that the MV is in range, thus making malicious anchors identification ineffective. So, it is desirable that for each iteration the MV follows a different path from the previous iterations. But, due to the limited number of paths repetitions are unavoidable. However, we note that repetition of a path should be infrequent and also the paths used for a given number of successive iterations should differ as much as possible. This makes it difficult for the adversary to predict the position of the MV.

In our scheme, for each iteration, the MV chooses a path from an ordered sequence of paths $\prod=\left\{\pi_{1}, \pi_{2}, \ldots, \pi_{m}\right\}$ that is pre-computed at the BS and stored in the MV. Each path $\pi_{i}, i=1, \ldots m$, is defined as a sequence $\pi_{i}=$ $\left\{B S, S_{k l}, S_{q r}, \ldots, B S\right\}$ of vertices of $G(V, E)$ where any two adjacent vertices in the sequence form an edge. For the first iteration, the MV chooses $\pi_{1}$, for the second $\pi_{2}$, and so on. When all the paths are used up, they are all available for selection again, and the procedure is repeated. For any two paths $\pi_{i}$ and $\pi_{j}$ in $\prod$ we define a score function, $\mathcal{F}\left(\pi_{i}, \pi_{j}\right)=\mid\left\{\left(S_{k l}, S_{q r}\right) \mid\left(S_{k l}, S_{q r}\right) \in \pi_{i}\right.$ and $\left.\pi_{j}\right\} \mid$. A smaller value of $\mathcal{F}\left(\pi_{i}, \pi_{j}\right)$ is desirable as it means that the difference between $\pi_{i}$ and $\pi_{j}$ is greater. The ordered sequence of paths $\Pi=\left\{\pi_{1}, \pi_{2}, \ldots, \pi_{m}\right\}$ is chosen by the BS such that for some given $p<m$ the function,

$$
\sum_{i=1}^{m-p} \sum_{j=i+1}^{i+p} \mathcal{F}\left(\pi_{i}, \pi_{j}\right)
$$

is minimized. That is, for a path $\pi_{i} \in \Pi$ the sum of the score functions corresponding to the next $p$ paths in $\Pi$ is minimized, hence these $p$ paths satisfy the desirable property of being as different from $\pi_{i}$ as possible, given a maximum possible choice of $m-1$ paths. This procedure is performed at the BS offline.

\section{B. Setup for Hypothesis Testing}

To test if an anchor is malicious, the MV performs hypothesis testing for the mean $(\mu)$ and the variance $\left(\sigma^{2}\right)$ of the location references. As described before, the measurement error is given by $\tilde{\mathbf{n}} \sim N\left(0, \sigma_{0}^{2}\right)$, where $\sigma_{0}^{2}$ is given by Equation 4. The estimate of the distance of an anchor $a_{i}$ from the MV can be modeled as $\hat{d}_{i}=d_{i} \cdot\left(1+\delta_{i}\right)$, where $\delta_{i}$ is the measurement error coefficient $\left(\sim N\left(0, \sigma_{0}^{2}\right)\right)$. In the event that $a_{i}$ is true, the calculated distance $d_{i}^{\text {calc }}$ satisfies Equation 6,

$$
d_{i}^{\text {calc }}=\left\|\mathbf{m}-\mathbf{a}_{\mathbf{i}}\right\|=d_{i},
$$

where $\mathbf{m}$ is the position of the MV. Hence the expression,

$$
\hat{d}_{i} / d_{i}^{\text {calc }}-1=\delta_{i},
$$

which is the coefficient for the measurement error. From the statistical perspective, given a sample of location references of an anchor $a_{i}$, if $a_{i}$ is true, the mean and the variance of the error should be close to the values 0 and $\sigma_{0}^{2}$ respectively. For a malicious anchor $a_{i}$, Equation 7 will not hold, as it may lie either about its distance or its position. As a result there would be a greater variance in the error coefficient $\delta_{i}$ and also a deviation in the mean $\mu_{i}$. We note that a sample variance greater than $\sigma_{0}^{2}$, or a shift in the sample mean, or both may be observed if $a_{i}$ lies. This is irrespective of how $a_{i}$ lies (position only, distance only, or both), as either $\hat{d}_{i}$, or $d_{i}^{\text {calc }}$, or both shall be affected by the lie. The tests we propose would be able to identify the malicious anchors using the above facts. For brevity, we illustrate the statistical tests for only the case where the anchors lie about the distance. However, the tests are applicable to identify anchors that are lying in the other ways mentioned.

From the location references obtained for each anchor $a_{i}$, we perform statistical hypothesis tests for the mean and the variance of the error coefficients. For more information about the theory of hypothesis testing we refer the reader to [12].

1) Hypothesis testing for mean: The hypothesis test for the sample mean $\mu$ is given by,

$$
H_{0}: \mu=0 \text { versus } H_{1}: \mu \neq 0 .
$$

We know that the mean of the distribution is $\mu=0$. A malicious anchor $a_{i}$ may lie such that the mean $\left(\mu_{i}\right)$ of the resultant error coefficients is non-zero. This malicious anchor can be identified if the null hypothesis of the above test is rejected. For the tests, we denote the probabilities for Type I error and Type II error by the standard variables $\alpha$ and $\beta$ [12]. The power of the test, defined as the probability of rejecting the null hypothesis $H_{0}$ when $H_{1}$ is true, is given by $1-\beta$. Hence, lower the value of $\beta$ higher is the power of the test. Given the sample mean $\bar{X}$, we can obtain the value 
of the test statistic, $Z_{0}=\frac{\bar{X}-0}{\sigma / \sqrt{(N)}}$, which is $\sim N(0,1)$. We would reject $H_{0}$ if $Z_{0}<-z_{\alpha / 2}$ or $Z_{0}>z_{\alpha / 2}$, where $z_{\alpha / 2}$ is the $100 \alpha / 2$ percentage point of $\sim N(0,1)$. And we would fail to reject $H_{0}$ otherwise. If $H_{0}$ is rejected it implies that the mean of the error coefficients of $a_{i}$ is in the critical region, which implies that $a_{i}$ is lying.

It is easy to see that with an increase in the sample size $N$ the accuracy of the test increases. However, this requires the MV to spend more time in each square $S_{x y}$. Hence, there exist trade-offs between the accuracy we require and the amount of time and energy required to attain such an accuracy. For given values of $\alpha, \beta$, and $\delta=\bar{X}-\mu$, the size of the sample $(N)$ required to produce the desired Type I and Type II errors is given by $N=\frac{\left(z_{\alpha}+z_{\beta}\right)^{2} \cdot \sigma^{2}}{\delta^{2}}$ [12]. The value of $\beta$ defines the probability of a false negative, higher the value of $\beta$ less powerful is the test, hence lower is the probability of rejecting $H_{0}$. For higher values of $\beta$, the tests can be performed for smaller values of $N$, but with the chance of increase in false negatives. However, we believe that false negative is not as serious as having false positive. A lying anchor that is not caught because of the lower power of the test owing to a small sample size can be caught subsequently with increasing sample size. On the other hand, false positive is highly undesirable as it may result in the anchor being incorrectly identified as malicious, hence resulting in its revocation. Thus in our tests we limit the Type I errors by using a high value for $\alpha$.

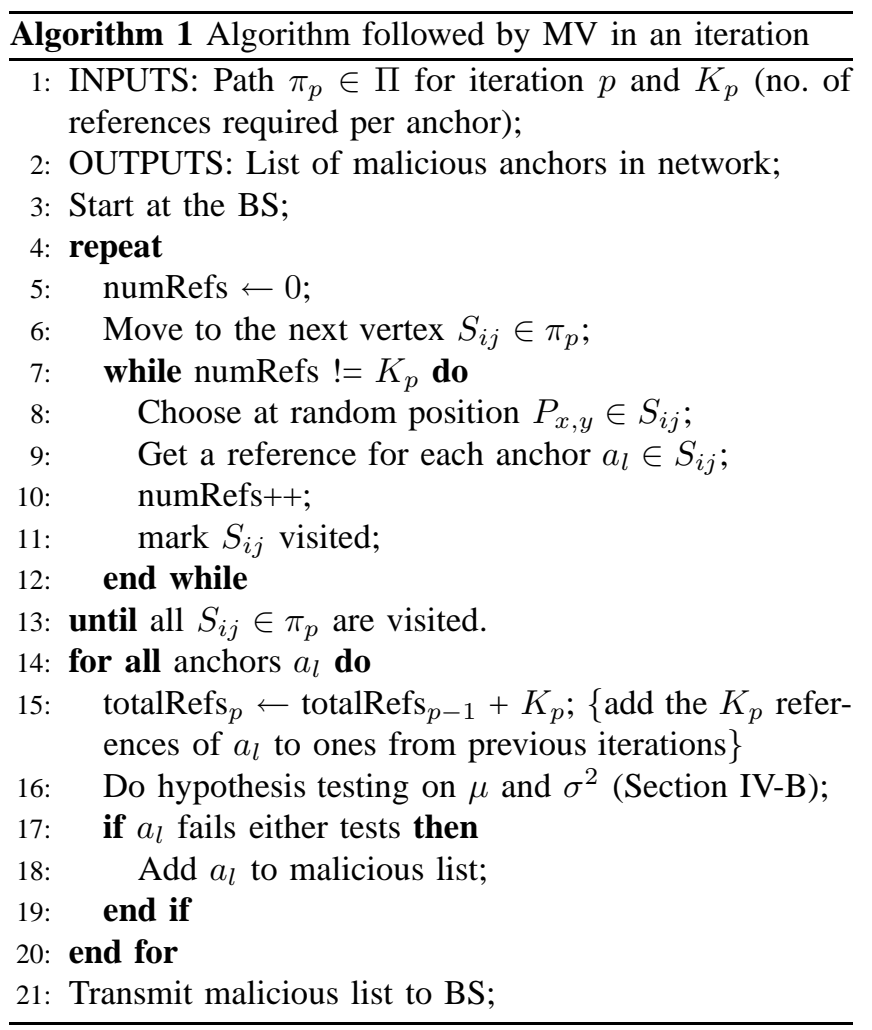

2) Hypothesis testing for the variance: At first, we present the motivation for performing hypothesis testing for the variance. Consider a malicious anchor $a_{i}$, let the distance estimate of $a_{i}$ from the MV be given as $d_{k}^{\prime}$ for the $k$ th reference in an iteration, and let the real distance be $d_{k}$. Let us consider that $a_{i}$ lies such that $d_{k}^{\prime}=d_{k} \cdot\left(1+(-1)^{k} \cdot \epsilon\right)$ for reference $k=1, \ldots, K$, where $\epsilon\left(>2 \cdot \delta_{\max }\right)$ is a constant error coefficient. In this case, despite $a_{i}$ lying by a significant amount, $\bar{X} \approx 0$, since the measurements alternate between two high extremes about $\mu=0$. Thus $a_{i}$ would not be caught despite lying significantly. This shortcoming can be remedied by testing the sample variance. The hypothesis testing on the sample variance $\sigma^{2}$ is given by,

$$
H_{0}: \sigma^{2}=\sigma_{0}^{2} \text { versus } H_{1}: \sigma^{2}>\sigma_{0}^{2} .
$$

In the above example, if we perform a hypothesis test for the variance, $a_{i}$ would be caught. The null hypothesis $H_{0}$ would be rejected as the variance of the sample obtained from $a_{i}$ would be $>>\sigma_{0}$.

For a sample of size $N$, we can define the test statistic $X_{0}^{2}=\frac{(N-1) \cdot \hat{s}^{2}}{\sigma_{0}^{2}}$, where $\hat{s}^{2}$ is the sample variance. $X_{0}^{2}$ follows the chi-square distribution $\chi^{2}$ with $N-1$ degrees of freedom denoted by $\chi_{0, n-1}^{2}$. Hence, the null hypothesis $H_{0}$ is rejected if $\chi_{0, n-1}^{2}>\chi_{\alpha, n-1}^{2}$ where $\chi_{\alpha, n-1}^{2}$ is the upper $100 \alpha$ percentage points of the $\chi_{n-1}^{2}$ distribution. If $H_{0}$ is rejected then $\hat{s}^{2}$ is in the critical region, hence the corresponding anchor is identified as malicious.

Algorithm 1 presents the operation of the MV in the network during a typical iteration. Once the MV gets the references from all the anchors, it performs the hypothesis test on the combined sample (from previous iterations and current iteration) to identify the malicious anchors.

The list of malicious anchors and their positions is broadcast by the BS as a revocation message in the network. The revocation process may also be initiated by the MV locally as soon as it identifies the malicious anchors in a square. The revocation messages for the SNs is authenticated by the BS using the $\mu$ TESLA mechanism.

\section{Simulation Results}

The WSN is deployed in a square field of dimensions $100 \times 100$ sq. units. This field is overlaid with a grid of squares of $20 \times 20$ sq. units. In each square, 10 anchors are deployed randomly. The transmission range of the anchors is set to $30 \mathrm{~m}$ and the location reference broadcast period is set to 1 second. The maximum error coefficient was chosen to be $\left|\delta_{\max }\right|=0.2$, the corresponding $\sigma_{0}^{2}=0.033$ and $\sigma=0.182 ; \alpha=0.01$ and $\beta=0.1$. Figure 2(a) shows the percentage of malicious and true anchors caught by the hypothesis test for $\mu$, given 3,5 , or 7 malicious anchors per square. The proportion of lie in the localization reference for each malicious anchor $a_{i}$ was chosen to be $\sim U[-0.2,0.4]$ thus having mean $\mu_{m}=0.1$. The first three curves represent 


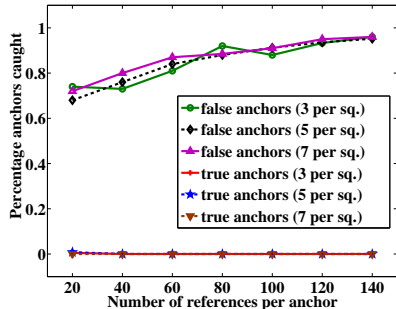

(a) Test of $\mu$

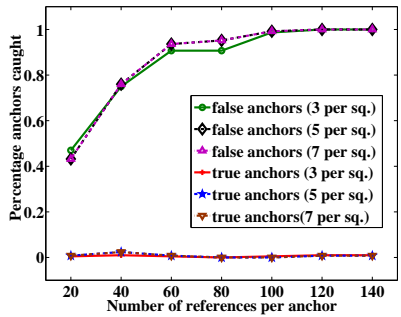

(c) Test for $\sigma^{2}$

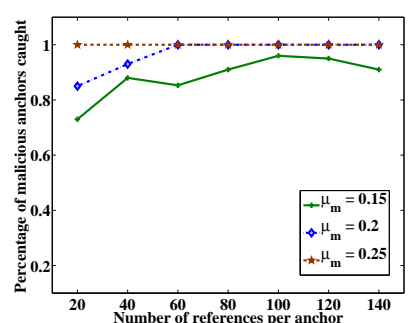

(b) Test for $\mu$

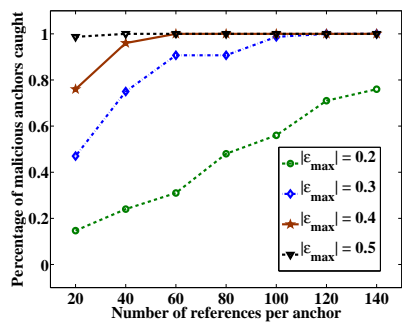

(d) Test for $\sigma^{2}$
Fig. 2. Simulation results. 2(a) and 2(c): Tests for $\mu$ and $\sigma^{2}$ with 3,5 , or 7 malicious anchors per square. 2(b) and 2(d): Percentage of malicious anchors caught for different proportions of lie.

the number of malicious anchors caught, while the bottom three curves represent the number of true anchors caught (false positives). Even with as few as 20 location references more than $70 \%$ of the malicious anchors are caught. As expected, with the increase in number of references more malicious anchors are caught. More than $90 \%$ malicious anchors were caught when the number of references were 60 or more. Also, the percentage of anchors caught is independent of the number of anchors in a square. We wish to point out that the percentage of false positives is close to 0 in our scheme. Figure 2(b) shows the percentage of malicious anchors caught for different values of the mean $\mu_{m}$ of the malicious anchors. As the anchors lie more, a greater number of the malicious anchors are caught with a lesser number of references. Even for a low value of $\mu_{m}=0.15$, our technique can catch more than $80 \%$ of the malicious anchors with as low as 40 location references. This shows that even with a small number of references the malicious anchors can be easily identified.

Figure 2(c) shows the results for the test for $\sigma^{2}$. Similar to the test for $\mu$, the false positives are low and the the number of malicious anchors caught increases with an increase in the number of references, with greater than $80 \%$ caught for the 60 or more references. Figure 2(d) shows the percentage of anchors caught with increasing lie coefficients. As the anchors lie more, more of them are caught. This is desirable for both the test for mean and variance, as anchors that lie more are more harmful for the localization process. Our scheme catches such anchors with high accuracy.

Hence our proposed technique can identify a large percentage of the malicious anchors in the network, thus improving the accuracy of SN localization.

\section{CONCLUSIONS AND FUTURE WORK}

In this paper, we propose a scheme that successfully identifies a large number of malicious anchors that may subvert the localization process in a WSN. In the future, we would like to find improved solutions for finding untraceable paths in the network and also study improvements in the energy requirements of the technique.

\section{REFERENCES}

[1] I. Akyildiz, W. Su, Y. Sankarasubramaniam, and E. Cayirci. Wireless sensor networks: A survey. Computer Networks, 38(4):393-422, 2002.

[2] Pratik Biswas, Tzu-Chen Lian, Ta-Chung Wang, and Yinyu Ye. Semidefi nite programming based algorithms for sensor network localization. ACM Transaction on Sensor Networks, 2(2):188-220, 2006.

[3] Xiuzhen Cheng, Andrew Thaeler, Guoliang Xue, and Dechang Chen. TPS: A time-based positioning scheme for outdoor wireless sensor networks. In Proceeding of Joint Conference of the IEEE Computer and Communications Societies (INFOCOM '04), volume 4, pages 2685-2696, 2004.

[4] L. Doherty, K. Pister, and L Ghaoui. Convex position estimation in wireless sensor networks. In Proceedings of the IEEE INFOCOM, pages 22-26, 2001.

[5] W. Du, L. Fang, and P. Ning. LAD: Localization anomaly detection for wireless sensor networks. In Proceedings of the 19th IEEE International Parallel and Distributed Processing Symposium (IPDPS), 2005.

[6] Tian He, Chengdu Huang, Brian M. Blum, John A. Stankovic, and Tarek F. Abdelzaher. Range-free localization and its impact on large scale sensor networks. Trans. on Embedded Computing Sys., 4(4):877-906, 2005.

[7] C. Karlof and D. Wagner. Secure routing in wireless sensor networks: Attacks and countermeasures. Elsevier's Ad Hoc Networks Journal, Special Issue on Sensor Network Applications and Protocols, 1(23):293-315, September 2003.

[8] L. Lazos and R. Poovendran. HiRLoc: High-resolution robust localization for wireless sensor networks. IEEE Journal on Selected Areas of Communications, 24(2):233-246, February 2006.

[9] L. Lazos, R. Poovendran, and S. Capkun. ROPE: Robust position estimation in wireless sensor networks. In Proceedings of Information Processing in Sensor Networks (IPSN), pages 324-331, 2005.

[10] Z. Li, W. Trappe, Y. Zhang, and B. Nath. Robust statistical methods for securing wireless localization in sensor networks. In Proceedings of Information Processing in Sensor Networks (IPSN), pages 91-98, 2005.

[11] D. Liu, P. Ning, and W. Du. Detecting malicious beacon nodes for secure location discovery in wireless sensor networks. In Proceedings of the 25th IEEE International Conference on Distributed Computing Systems (ICDCS), pages 609-619, 2005.

[12] D. Montgomery and G. Runger. Applied Statistics and Probability for Engineers. John Wiley and Sons Inc., 2002.

[13] D. Niculescu and B. Nath. Error characteristics of ad hoc positioning systems (APS). In Proceeding of ACM MobiHoc, 2004.

[14] A. Perrig, R. Canetti, D. Tygar, and D. Song. The tesla broadcast authentication protocol. Cryptobytes, 5(2):2-13, 2002.

[15] A. Savvides, C. Hans, and M. Srivastava. Dynamic fi ne-grained localization in ad-hoc networks of sensors. In Proceeding of ACM MobiCom, pages 166-179, 2001.

[16] S. Čapkun and J. Hubaux. Secure positioning in wireless networks. IEEE Journal on Selected Areas of Communications, 24(2):221-232, February 2006.

[17] A. Wood and J. Stankovic. Denial of service in sensor networks. IEEE Computer, 35(10):54-62, oct 2002. 